\title{
Desafios da Formação para o Trabalho Interprofissional no Contexto da Reabilitação
}

\author{
Challenges of Training for Interprofessional Work in Rehabilitation \\ Desafios de la Formación para el Trabajo Interprofesional en el Contexto de la \\ Rehabilitación
}

José Gutembergue de Vasconcelos Bezerra ${ }^{1}$

Cristina Camelo de Azevedo ${ }^{2}$

Josineide Francisco Sampaio ${ }^{3}$

\section{Resumo}

Objetivo: Compreender a dinâmica da formação em saúde, por meio da preceptoria, a partir dos princípios da educação interprofissional em um centro especializado em reabilitação de uma universidade pública brasileira. Método: Foi desenvolvida uma pesquisa descritiva, exploratória, com abordagem qualitativa, em que foram entrevistados preceptores dos três cursos visando identificar ações interprofissionais nas atividades de estágio curricular e visibilizar potenciais barreiras e elementos facilitadores desse processo. As práticas discursivas e produção de sentidos fundamentaram a pesquisa e a elaboração de mapas dialógicos serviu de instrumento de análise das falas dos entrevistados. Resultados: Constatou-se que não existem atividades interprofissionais estabelecidas nos estágios supervisionados e, embora haja

${ }^{1}$ Terapeuta ocupacional graduado pela UFPE. Pós-graduação em Neuropsicologia. Mestrado Profissional em Ensino na Saúde pela Universidade Federal de Alagoas. Terapeuta ocupacional do HUPAA/UFAL/EBSERH e CER III PAM Salgadinho - Maceió AL. Endereço completo: Av. Brigadeiro Eduardo Gomes. No 1205. Cruz das Almas. 57038-230. Maceió - AL, Brasil. E-mail: zebergue1978@gmail.com

${ }^{2}$ Psicóloga. Doutorado em Saúde Pública pela ENSP/FIOCRUZ. Professora do Instituto de Psicologia e do Mestrado Profissional em Ensino na Saúde da Universidade Federal de Alagoas

${ }^{3}$ Socióloga. Doutorado em Saúde Pública pela ENSP/FIOCRUZ. Professora da Faculdade de Medicina e do Mestrado Profissional em Ensino na Saúde da Universidade Federal de Alagoas

Recebido: set./2017 - Aceito: mar./2018. 
o entendimento de que a reabilitação se potencializa com as práticas colaborativas, não se reconhece $\mathrm{o}$ interesse profissional ou institucional, em adotar essa metodologia de trabalho. Conclusão: $O$ desafio de legitimar a interprofissionalidade no cotidiano das equipes de reabilitação e na prática educacional através dos estágios supervisionados, depende bem mais do interesse e envolvimento individuais do que do apoio institucional ou mobilização de outros membros da equipe.

\section{Descritores: \\ Preceptoria; \\ Reabilitação; \\ Relações \\ Interprofissionais.}

\section{Abstract}

Objective: Understand the dynamics of health training, through preceptory, based on the principles of interprofessional education in a center specialized in rehabilitation of a brazilian public university. Methods: It was developed a descriptive and exploratory research with qualitative approach in which were interviewed preceptors of the three courses aiming to identify interprofessional actions in the activities of curricular internship and to visualize potential barriers and facilitating elements of that process. The discursive practices and sense production grounded the research and the elaboration of dialogical maps served as an instrument for the analysis of the interviewees' speeches. Results: It was observed that there are no interprofessional activities established in the supervised stages, and although there is an understanding that rehabilitation is enhanced by collaborative practices, it is not recognized the professional or institutional interest in adopting this work methodology. Conclusion: The challenge of legitimizing interprofessional practices in the daily life of rehabilitation teams and in educational practice through supervised internship depends more on individual interest and involvement than on institutional support or mobilization of other team members.

\section{Descriptors:}

Preceptorship;

Rehabilitation;

Interprofessional

Relations.

\section{Resumen}

Objetivo: Comprender la dinámica de la formación en salud, a través de la preceptoria, a partir de los principios de la educación interprofesional en un 
centro especializado en rehabilitación de una universidad pública brasileña. Métodos: Fue desarrollada una investigación descriptiva, exploratoria, con enfoque cualitativo, en que fueron entrevistados preceptores de los tres cursos, para identificar acciones interprofesionales en las actividades de prácticas curriculares y visibilizar potenciales barreras asi como elementos facilitadores de este proceso. Las prácticas discursivas y producción de sentidos fundamentaron la investigación, y la elaboración de mapas dialógicos sirvieron de instrumento de análisis de las charlas de los entrevistados. Resultados: Se constató que no existen actividades interprofesionales establecidas en las prácticas supervisadas y, aunque existe la comprensión de que la rehabilitación se potencializa con las prácticas de colaboración, no se reconoce el interés profesional o institucional en adoptar esa metodología de trabajo. Conclusión: El desafio de legitimar la interprofesionalidad en el cotidiano de los equipos de rehabilitación y en la práctica educativa a través de las prácticas supervisadas, depende mucho más del interés y de la participación individual que del apoyo institucional o de la movilización de otros miembros del equipo.

DESCRIPTORES:

Preceptoría;

Rehabilitación;

Relaciones

Interprofesionales.

Descriptores:

Rehabilitación;

Interprofesionales.

\section{Introdução}

A atenção em saúde demanda que ações integradas de diferentes profissionais sejam direcionadas à pessoa cuidada conforme suas necessidades e especificidades, o que se entende como trabalho em equipe.

A interprofissionalidade se apresenta, portanto, como alternativa à multiplicidade dos saberes profissionais desarticulados e a educação interprofissional como uma modalidade de formação em saúde que promove o trabalho em equipe integrado e colaborativo entre profissionais de diferentes áreas com foco nas necessidades de saúde dos usuários ${ }^{(1,2)}$.

Em 2010, a Organização Mundial de Saúde lançou o Marco para Ação em Educação Interprofissional e Prática Colaborativa ${ }^{(3)}$, que vem servindo desde então como referência para pesquisadores da saúde e da educação ressaltando o diferencial que o trabalho desenvolvido em equipe representa 
$(2,4,5,6)$. Isso nos leva a pensar na

necessidade da formação em saúde com oferta de práticas coletivas e interprofissionais.

Dessa maneira, a participação dos discentes em atividades de estágio supervisionado fundamentadas na educação interprofissional em saúde poderia funcionar como estratégia favorecedora de aprendizagens compartilhadas, oportunizando a cooperação para o exercício permanente do diálogo ${ }^{(7)}$.

Levando-se em conta o potencial de ganho de qualidade de vida dos usuários resultantes do trabalho das equipes de reabilitação, compreende-se que esse campo de cuidados em saúde exige um encontro harmonioso entre os diversos saberes e práticas que $\mathrm{o}$ compõe.

Essa pesquisa objetivou compreender a dinâmica da formação em saúde, por meio da preceptoria, a partir dos princípios da educação interprofissional num centro especializado em reabilitação de uma universidade pública brasileira, buscando visibilizar suas barreiras e elementos facilitadores, reconhecendo os centros de reabilitação como cenários multiplicadores da interprofissionalidade.

\section{Método}

\section{Tipo de Estudo}

Esse trabalho consiste em um estudo de abordagem qualitativa, descritivo e exploratório, iniciado após aprovação pelo Comitê de Ética na Pesquisa da Universidade Federal de Alagoas através do parecer $\mathrm{n}^{\mathrm{o}}$ 1.455.549 e não apresenta nenhum tipo de conflito de interesse. Foi desenvolvido no Centro Especializado em Reabilitação de uma universidade pública brasileira, direcionado aos cursos de fisioterapia, fonoaudiologia e terapia ocupacional, por serem os que realizam, neste centro, atividades de preceptoria.

\section{Coleta de Dados}

$\mathrm{O}$ instrumento utilizado para a produção das informações foi a entrevista semiestruturada.

\section{Participantes da Pesquisa}

Foram selecionados 6 profissionais através de sorteio, 2 relativos a cada curso, por desenvolverem atividades de preceptoria no centro especializado e não exercerem concomitantemente 
atividade de gestão acadêmica de ensino.

\section{Análise dos Dados}

Como ferramentas de análise foram utilizados os mapas dialógicos e os repertórios linguísticos dos discursos, que fazem parte do aporte teórico utilizado, denominado Práticas Discursivas e Produção de Sentidos ${ }^{(8)}$.

Para a elaboração dos mapas dialógicos foi realizada a transcrição literal das entrevistas. A observação e leitura das falas e sentidos produzidos através dos diálogos definiram as categorias analíticas, passando a constituírem os vetores centrais das discussões.

Assim, sob o tema "Trabalho e Educação Interprofissional em Saúde no Centro Especializado em Reabilitação da Universidade Estadual de Ciências da Saúde de Alagoas", três categorias foram representadas nos mapas dialógicos:

1. Concepções sobre educação interprofissional em saúde e interprofissionalidade;
2. Aspectos facilitadores e dificultadores de ações interprofissionais;

3. A instituição como promotora do trabalho e educação interprofissional em saúde.

\section{Resultados e Discussão}

$\begin{array}{lll}\text { Trabalho } & \text { e } & \text { Educação }\end{array}$ Interprofissional em Saúde no Centro Especializado em Reabilitação da Universidade Estadual de Ciências da Saúde de Alagoas

Concepções dos Preceptores sobre Trabalho $\quad$ e $\quad$ Educação Interprofissional em Saúde

Sobre este item, foram destacados sentidos e repertórios derivados das falas constantes dos mapas dialógicos (Quadro 1).

Os repertórios surgidos nas entrevistas com relação à compreensão dos preceptores sobre o trabalho e a educação interprofissionais em saúde exteriorizam, em princípio, duas situações contraditórias quanto a teoria e a prática da interprofissionalidade. 


\section{Quadro 1:}

\begin{tabular}{|c|c|}
\hline & REPERTÓRIOS \\
\hline $\begin{array}{l}\text { Compreensão do conceito de } \\
\text { interprofissionalidade }\end{array}$ & $\begin{array}{l}\text { Conceito intercruzado } \\
\text { Termo novo } \\
\text { Mesma lógica de interdisciplinaridade }\end{array}$ \\
\hline $\begin{array}{l}\text { Entendimento da prática } \\
\text { interprofissional }\end{array}$ & $\begin{array}{l}\text { Conhecer a atividade do outro } \\
\text { Trabalhar juntos } \\
\text { Trocar saberes } \\
\text { Trabalho em equipe } \\
\text { Trabalho que reúne profissões } \\
\text { Juntar profissões } \\
\text { Ver o paciente como um todo } \\
\text { Conjunto de intervenções para a } \\
\text { reabilitação }\end{array}$ \\
\hline
\end{tabular}

Para alguns entrevistados, não há uma definição conceitual clara sobre o tema - havendo inclusive indistinção com relação à interdisciplinaridade; mas apesar disso, ao exemplificarem como o trabalho interprofissional se expressaria no cotidiano, constata-se que não há incompatibilidade entre ambas.

Isso se explica ao identificarmos nas falas uma aproximação com a ideia de um trabalho desenvolvido em sintonia, integrado entre profissionais de diferentes áreas com um objetivo em comum. partes como a gente costuma ver. (Entrevistado 2)

Eu imagino que tenha a ver com Interdisciplinaridade... não sei... seria você trabalhar de forma conjunta com profissionais de outra área pensando num objetivo comum pra ... um paciente enfim. (Entrevistado 3).

Apesar de se perceber que os conceitos entre interdisciplinaridade e interprofissionalidade se confundem e, às vezes, não se diferenciam, pode-se apreender dos diálogos dos preceptores, que há, também, uma aproximação com o que se constitui como trabalho interprofissional. Sobre a relação entre a interdisciplinaridade e $\mathrm{a}$ interprofissionalidade, entende-se que:

Para que haja interprofissionalidade, necessária seria, então, uma etapa anterior, qual seja, a interdisciplinaridade, considerando dever a prática pautar-se por saberes. Se a integração de saberes se efetiva na interdisciplinaridade, então podemos considerar como sendo esta uma condição para a 
interprofissionalidade. Desta forma, entendemos estar a interdisciplinaridade contida na interprofissionalidade ${ }^{(4)}$.

Esta reflexão remete ao discurso trazido por um dos entrevistados:

\begin{abstract}
Eu vejo interdisciplinar como algo mais amplo em termos de conhecimentos e de áreas... amplas áreas, não sei se é isso! E interprofissional parece que é algo mais próximo da prática... interprofissional esteja mais relacionado a sua prática profissional $e$ o interdisciplinar talvez a áreas de conhecimento que se interpõem. (Entrevistado 3)
\end{abstract}

Ao considerarmos a complexidade e a abrangência do campo da reabilitação, bem como o seu potencial de impacto na vida das pessoas, a proposta de se trabalhar interprofissionalmente, em princípio demandaria da equipe uma compreensão clara sobre seu conceito, sobretudo diferenciando-o do conceito da interdisciplinaridade.

Porém, embora haja pouca clareza entre os preceptores sobre a diferença conceitual de ambas, o entendimento do que seria uma prática interdisciplinar se aproxima com o que de fato se caracteriza como interprofissionalidade. Assim, poder-se-ia afirmar que a sua não aplicação, não se justifica pela carência de conhecimento sobre o tema.
Por vezes, os preceptores interpretam o entrosamento entre colegas da mesma profissão, ainda que de áreas de atenção diferentes, entre reabilitação do adulto e da criança, como interprofissionalidade:

Eu tenho uma experiência também de
interprofissionalidade de setores da
fisioterapia... por exemplo o meu setor
é de adulto, e a gente agora recebe
pacientes da pediatria ... foi
interessante porque para onde vão
esses meninos da pediatria quando
ficam jovens?... a gente começou a
criar uma ligação com a professora da
pediatria ... e a partir de uma triagem
eles vêm para a gente e começamos a
atender, então hár uma
interprofissionalidade também.
(Entrevistado 6).

Disso pode-se apreender, no mínimo, duas vertentes de interpretação: a primeira poderia ser entendida como uma resistência ao trabalho com outras profissões, persistindo o olhar especializado, mas passando a ideia de que, dessa forma, estaria caracterizada uma atividade interprofissional, quando, na verdade, poder-se-ia concebê-la apenas como 'intraprofissional'; e a segunda, que se mostra mais pertinente, sugere que, independente de tratar-se da mesma profissão, há disposição de ambos os profissionais para a resolubilidade através do diálogo, da integração e da prática colaborativa. 
Do mesmo modo como existem problemas decorrentes da falta de integração/interação entre os profissionais, a mudança para um trabalho interprofissional dialogado e participativo trará à tona novos problemas que não podem nem devem ser desencorajadores, mas entendidos como componentes de um processo que gerará muito mais ganhos que perdas, mais crescimento que entraves, e que o aprendizado será fruto deste enfrentamento.

A expressão disso pode ser percebido a partir dos resultados das práticas colaborativas entre as diferentes profissões que compartilham de um mesmo objetivo, reduzindo a influência da superespecialização do conhecimento e da técnica. Desse modo, os pontos de divergência, a exemplo da defesa acirrada por áreas do saber, podem interferir menos na dinâmica das relações profisssionais.

\section{Fatores Facilitadores e Dificultadores de Ações Interprofissionais}

Parte do conteúdo elaborado nas entrevistas inclinou-se a discorrer sobre os componentes favorecedores $\mathrm{e}$ limitadores da prática interprofissional, mas também chama atenção o fato de que, em algum momento, os próprios preceptores se auto referenciaram nessas reflexões:

\begin{abstract}
O que custa eu ir lá falar com o fisioterapeuta que atende o mesmo paciente que eu? Eu acho que eu poderia buscar mais o conhecimento do outro, estar indo mais atrás do outro, coisa que eu não faço. (Entrevistado 2)

Talvez seja um problema meu também... eu não bati lá na porta para falar com o profissional, eu acho que é uma responsabilidade que é minha de certa forma. (Entrevistado 5)
\end{abstract}

Destaca-se a produção de sentidos relativos aos fatores facilitadores e dificultadores da interprofissionalidade, bem como a auto avaliação dos preceptores sobre a interprofissionalidade no contexto da reabilitação (Quadro 2).

Apesar da obviedade do discurso das vantagens do cuidado conjunto, integrado e articulado, sobretudo num ambiente de formação, ocorre claramente a contradição das ações isoladas, não dialogadas e não pactuadas.

Várias são as justificativas que tentam pôr luz ao fato, e todas exercem, em alguma medida, influência nas atitudes individuais ou grupais, intra e interprofissões, sobre as normas, condutas e métodos de atendimento; todavia há de se reconhecer que, caso se pretenda implementar um ambiente que 
promova a educação interprofissional, tão ou mais importante que o incentivo da gestão - garantindo os meios, recursos e estratégias necessários - é o desejo individual de cada profissional, em maior ou menor grau, que determinará o sucesso ou o fracasso desse projeto.

\section{Quadro 2:}

\begin{tabular}{c|l} 
& \multicolumn{1}{c}{ REPERTÓRIOS } \\
\hline \multirow{2}{*}{$\begin{array}{c}\text { Fatores facilitadores } \\
\text { de ações interprofissionais }\end{array}$} & Vontade \\
& $\begin{array}{l}\text { Amizade } \\
\text { Abertura } \\
\text { Troca }\end{array}$ \\
\hline \multirow{3}{*}{$\begin{array}{c}\text { Fatores dificultadores de ações } \\
\text { interprofissionais }\end{array}$} & Falta de querer se implicar \\
& Trabalhar individualmente é mais fácil \\
Auto avaliação dos preceptores & Inseguranças \\
\hline & Medo de estar invadindo a profissão do \\
& outro \\
& Receio de ser avaliado \\
& Não querer se expor \\
& Dialogar é desconstruir
\end{tabular}

Por outro lado, ao serem questionados sobre as razões do desinteresse da equipe de reabilitação pelo trabalho interprofissional, os preceptores levantam algumas hipóteses que podem explicar os motivos dessa relutância, como o medo de invasão do espaço ou atribuições do outro, muito comum no universo da reabilitação; ou insegurança de, numa situação de atendimento ou avaliação conjunta, sentirem-se expostos ou avaliados.

Quando questionados sobre o principal fator para a não existência de um trabalho integrado com maior comunicação entre os profissionais, algumas falas se destacaram por, mesmo considerando as carências estruturais e organizacionais do serviço, apontarem que a falta de vontade dos profissionais em trabalhar em conjunto é o principal fator responsável pela desarticulação da equipe.

\section{Sinceramente eu acho que as pessoas não querem! Elas não querem...é mais fácil você trabalhar individualmente do que você trabalhar em conjunto. (Entrevistado 3)}

$$
\text { Considera-se que condições }
$$
favoráveis para um bom relacionamento e trabalho interprofissionais são necessárias, mas não suficientes, considerando dependerem estas também da mobilização e disposição de cada sujeito em particular ${ }^{(4)}$.

...estão todos tão perto fisicamente, o
que custa eu subir e falar com a fono 
sobre um paciente que nós atendemos? (Entrevistado 2)

$\mathrm{Na}$ maioria das falas dos entrevistados, ao mesmo tempo em que não se reconhece a existência de práticas interprofissionais, se considera a intenção ou iniciativas individuais de alguns professores, nessa direção.

Existe uma vontade. Eu acho que a equipe já vislumbra essa possibilidade, existe uma vontade... alguns profissionais já saem para ir buscar o outro... acho que isso já é um avanço. (Entrevistado 1)

O que seriam as práticas colaborativas, senão a convergência do querer e do fazer focados num objetivo comum? Quando questionados se, acaso todas estas demandas fossem atendidas e as supostas causas de sua inexistência sanadas, isso por si só garantiria a interprofissionalidade e, por consequência, a educação interprofissional, a resposta unânime foi “não!”. E poucos souberam justificar o porquê, mas um dos professores manifestou que o problema se estabelece na deficiência da formação em oportunizar desde os primeiros semestres acadêmicos, estudos e vivências em educação interprofissional.

A formação muito organicista dificulta esse tipo de conduta. Não existe (interprofissionalidade)! Pelo menos na clínica em que eu atuo, não! ...nunca vi! Seria muito necessário! Isso envolve antecedentes! Formação! (Entrevistado 4)

Em princípio, as práticas de estágio supervisionado dos cursos funcionavam em prédios contíguos, sem comunicação direta ou intercâmbio de práticas de ensino e atendimento. Após a habilitação do Centro de reabilitação, a sua dinâmica de serviços ambulatoriais de reabilitação seguiu a mesma tendência, atuando multidisciplinarmente com interação entre as profissões ainda incipiente. Assim, preceptores e discentes desenvolvem suas atividades majoritariamente de forma isolada, sem o exercício do diálogo interprofissional.

A questão da formação acadêmica com base na integração dos cursos vem ocorrendo na Universidade Estadual de Ciências da Saúde de Alagoas desde a elaboração das novas matrizes curriculares implantadas em 2014, cabendo considerar que agregar turmas de diferentes cursos em módulos não garante, por si só, a integração entre os mesmos; mas deve-se destacar que cria um ambiente favorável a isso e o professor terá um papel essencial nessa tarefa como direcionador e distensionador de eventuais conflitos previsíveis entre os diferentes cursos, 
dada a tradição de formação por especialidades e competitividade entre classes.

\section{A Instituição como Promotora do} Trabalho e Educação Interprofissional em Saúde

A temática da formação interprofissional suscita nos preceptores falas sobre as expectativas de experiências de integração com profissionais de outras áreas vivenciadas pelos estudantes:

\begin{abstract}
Acho que só teriam a ganhar com essa troca, essa interação de um profissional com o outro, eu só vejo pontos positivos e sairiam os discentes da universidade com essa visão da interação com outros profissionais. (Entrevistado 2)
\end{abstract}

Os sentidos produzidos sinalizam fatores facilitadores dessa prática, embora entendendo, num contexto de integração ensino-serviço, a promoção das ações incentivadoras como responsabilidade exclusiva da gestão do Centro de reabilitação (Quadro 3).

\begin{tabular}{|c|c|}
\hline & REPERTÓRIOS \\
\hline Responsabilização da gestão & $\begin{array}{l}\text { A instituição tem que se preparar } \\
\text { Demora em tomar as decisões } \\
\text { Prédios separados } \\
\text { Dificuldades com prontuários } \\
\text { Centralizado na gestão } \\
\text { Não dá para ter vontade sozinho }\end{array}$ \\
\hline $\begin{array}{l}\text { Possíveis ações incentivadoras } \\
\text { de TIP e EIP através da gestão }\end{array}$ & $\begin{array}{l}\text { Horário para a avaliação global } \\
\text { Discussões de caso com regularidade } \\
\text { Triagem interprofissional } \\
\text { Sensibilizações } \\
\text { Planejamentos participativos } \\
\text { Capacitações } \\
\text { Estágios integrados } \\
\text { Planejamento terapêutico conjunto } \\
\text { Reforma estrutural }\end{array}$ \\
\hline
\end{tabular}

No conjunto de depoimentos dos preceptores, foi atribuída à gestão a responsabilidade quanto à implantação da prática interprofissional no serviço e apontadas a reforma dos espaços e estrutura física, a implantação de prontuários únicos e informatizados, a necessidade de definição de agendas de encontros e reuniões de discussão de casos e oficinas de capacitação e sensibilização sobre a interprofissionalidade, como forma de legitimá-la, de efetivá-la. 
Há de se ressaltar que o trabalho interprofissional e a prática colaborativa exigem muito mais que mudanças e adaptações de espaços, rotinas e técnicas terapêuticas de atendimento; entretanto, também se considera que:

A colaboração entre profissionais que queiram trabalhar juntos em prol da melhor qualidade da atenção à saúde inclui aspectos pessoais, mas depende de dimensões da organização na qual os trabalhadores estão e na articulação desta organização com o sistema ${ }^{(9)}$.

O ponto de equilíbrio poderia vir da junção de esforços de todos os componentes na busca pela efetivação de práticas interprofissionais, inclusive dos próprios acadêmicos, atuando como mobilizadores de ações nesse sentido.

Sobre a motivação da equipe para as práticas colaborativas, entende-se que um líder de projeto em educação interprofissional em saúde atuaria como condutor de ações e atividades do grupo voltadas para este fim:

Organizadores precisam providenciar reuniões regulares e considerar todas as perspectivas e também requerem habilidades interprofissionais $[\ldots]$ e as organizações precisam constantemente avaliar, revisar e discutir a EIP dentro da organização para lembrar todos os membros do grupo a respeito do objetivo geral da EIP, que é a prática interprofissional ${ }^{(10)}$.

Mas o autor também reconhece a complexidade em manter a educação interprofissional na rotina dos trabalhos, por exigir bom nível de diálogo entre a equipe e entusiasmo constante na realização das atividades assistenciais/pedagógicas.

Sobre isso, uma das propostas trazidas pelos entrevistados diz respeito aos ‘estágios integrados':

Acho que talvez também pudesse existir esse espaço de um estágio integrado mesmo, um estágio onde eu pudesse ter um trabalho entre vários profissionais, acho que isso é possivel! Se é possivel na saúde pública, na saúde mental. (Entrevistado 5)

Deve-se considerar, no entanto, que projetos de estágios integrados competem muito mais às coordenações de estágios supervisionados dos cursos, articulando estratégias para este fim. À gestão do centro de reabilitação caberia oportunizar condições e meios, apoiando estas iniciativas. Uma alternativa apresentada nas entrevistas poderia funcionar como experiência para implantar práticas interprofissionais com a participação dos estudantes:

Para favorecer esses momentos de aprendizado, porque não, alunos dos cursos que existem na universidade 
avaliarem em conjunto? Uma triagem interprofissional (Entrevistado 4).

A educação interprofissional na reabilitação seria favorecida através da participação dos acadêmicos junto aos profissionais do Centro na elaboração dos objetivos de intervenção e plano de atendimento de cada paciente em particular.

Compreende-se, portanto, que a implementação das ações interprofissionais podem ser pontuais em princípio, não devendo ocorrer de vez, por determinação ou imposição, mas a partir do movimento e interesse de alguns, que vão abrindo caminhos para sua instalação gradativa e espontânea, até que possa ser instituída como metodologia de trabalho.

\section{Conclusão}

Embora represente um avanço que o tema da interprofissionalidade esteja textualmente apontado nos projetos pedagógicos dos cursos analisados, o desafio parece ser como efetivá-la na prática educacional, como legitimá-la no cotidiano do Centro de reabilitação que atua como unidade formadora por intermédio da preceptoria.

Um dos caminhos a se percorrer para este fim certamente é o de identificar os fatores que impedem que a proposta de trabalho interprofissional seja implementada para, a partir disso, traçar as estratégias necessárias rumo a este novo modelo de atenção à saúde $\mathrm{e}$ de ensino.

A reflexão sobre a temática do trabalho e da educação interprofissional na saúde e reabilitação conduz a uma constatação particular de que sua implementação se sustenta muito mais na autopercepção dos profissionais sobre seus limites, desejos e anseios, do que em fatores externos a si, ou seja, dependentes do outro, da gestão, ou dos métodos de trabalho vigentes, como apontam os dizeres mais rápidos $\mathrm{e}$ pouco refletidos, já que todos estes são secundários àquele e sinalizam que é sobretudo no movimento de dentro para fora, do individual para o coletivo, que se materializam as mudanças.

Mas, para que se propicie aos acadêmicos um ambiente educacional voltado para as práticas colaborativas, as práticas de trabalho interprofissional devem estar consolidadas.

Quanto a isso, a experiência atual do Centro de reabilitação, trazida pelos entrevistados, nos mostra duas situações; a primeira nos revela que há resistências entre alguns profissionais ao estabelecimento de rotinas de trabalho integrado e interprofissional - 
muito embora essas impressões se deem em relação ao outro; a segunda mostra que em uma autoavaliação, os preceptores se percebem favoráveis a esta forma de trabalho.

\section{Referências}

1. Rossit R A S, Batista S H, Batista N A. Formação interprofissional em saúde: percepção de egressos de cursos de graduação da UNIFESP-Baixada Santista. In: Encontro Nacional de Pesquisa em Educação em Ciências. IX ENPEC Águas de Lindóia, p. 0107. 2013. Disponivel em: http://www.nutes.ufrj.br/abrapec/ixenp ec/atas/resumos/R1395-1.pdf

2. Peduzzi M, Norman IJ, Germani ACCG, Silva JAM, Souza GC. Educação interprofissional: formação de profissionais de saúde para o trabalho em equipe com foco nos usuários. Rev Esc Enferm. USP [periódico na Internet]. 2013 [citado 2016 mar. 01]; 47(4):977-83. Disponível em: http://www.revistas.usp.br/reeusp/articl e/view/78051

3. World Health Organization (WHO). Framework for action on interprofessional education and collaborative practice. Geneva: WHO; 2010.

4. Ellery A E L. Interprofissionalidade na Estratégia Saúde da Família: condições de possibilidade para a integração de saberes e a colaboração interprofissional. Interface (Botucatu) [periódico na Internet]. 2014 [citado 2015 out. 10]; 18(48):213-14. Disponível em: http://www.scielo.br/scielo.php?script $=$ sci_arttext\&pid=S141432832014000100213.
5. Peduzzi M. Trabalho e educação na saúde: ampliação da abordagem de recursos humanos. Ciênc Saúde Coletiva [periódico na Internet]. 2013 [citado 2014 out. 12];18(6):1539-41. Disponível em: http://www.scielo.br/pdf/csc/v18n6/05. pdf.

6. Aguilar-da-Silva RH, Scapin LT, Batista NA. Avaliação da formação interprofissional no ensino superior em saúde: aspectos da colaboração e do trabalho em equipe. Avaliação (Campinas) [periódico na Internet]. 2011[citado 2016 mar. 10];16(1):16582. Disponível em: http://www.scielo.br/pdf/aval/v16n1/v $16 \mathrm{n} 1 \mathrm{a} 09$.

7. Batista NA. Educação interprofissional em Saúde: concepções e práticas. Cad FNEPAS [periódico na Internet]. 2012 [citado 2016 mar. 10];2(2):25-8. Disponível em:

http://www.fnepas.org.br/artigos_cader no/v2/educacao_interprofissional.pdf.

8. Spink M J (org.). Práticas discursivas e produções de sentido no cotidiano. $2^{a}$ ed. São Paulo: Ed. Cortez, 2000 .

9. Germani ACC, Oliver FC, Rocha EF, Carvalho YM, Peduzzi M, Sangaleti C. Aprender a trabalhar juntos: desafios no ensino da prática interprofissional e colaborativa [Internet]. 2010 [citado 2017 dez. 11]. Disponível em: http://www.convibra.org/upload/paper/ 2013/59/2013_59_6391.pdf.

10. Reeves S. Porque precisamos da educação interprofissional para um cuidado efetivo e seguro. Interface (Botucatu) [periódico na Internet]. 2016 [citado 2016 dez. 01]; 20(56):185-96. Disponível em: http://www.scielo.br/pdf/icse/v20n56/p t_1807-5762-icse-20-56-0185.pdf. 